\title{
Some fixed point theorems on nonlinear contractive mappings in Hilbert space
}

\author{
Namana Seshagiri Rao and Karusala Kalyani \\ Communicated by Ljubisa Kocinac
}

\begin{abstract}
The purpose of this paper is to establish the existence and uniqueness of a common fixed point for a pair of continuous self mappings over a closed subset of Hilbert space satisfying certain nonlinear rational type contraction condition. Further this result is extended and generalized for some positive integers powers of a pair of continuous self mappings and then is devolved for a sequence of continuous self mappings in the Hilbert space. Our results generalize and extend many well known results in the literature.
\end{abstract}

Keywords. Hilbert space, closed subset, Cauchy sequence, completeness.

2010 Mathematics Subject Classification. 40A05, 47H10, 54H25.

\section{Introduction}

The existence and uniqueness of a fixed point was given by Banach [2] in 1922, which was acclaimed as Banach contraction principle and plays an important role in the development of various results connected with Fixed point Theory and Approximation Theory. The Banach fixed point theorem or the contraction principle concerns certain mappings of a complete metric space into itself. It lays down conditions; sufficient for the existence and uniqueness of a fixed point. Besides, this famous classical theorem gives an iteration process through which we can obtain better approximation to the fixed point. Banach's fixed point theorem has rendered a key role in solving systems of linear algebraic equations involving iteration process. Iteration procedures are used in nearly every branch of applied mathematics, convergence proof and also in estimating the process of errors, very often by an application of Banach's fixed point theorem.

Later lots of improvements have been done by many authors such as Kannan [17] investigated the extension of Banach fixed point theorem by removing the completeness of the space with different sufficient conditions. Chatterji [4,5] considered in his work by taking various contraction conditions for self mappings in metric space which are derived from the theorem of Zamfirescu [32] taking suitable combinations of positive constants. Rational type of contractions leads to obtain a unique fixed point in complete metric space was investigated by Dass 
and Gupta [7]. Fisher [8] developed the approach of Kannan [16] and proved analogous results involving two mappings on a complete metric space. A generalization of Banach fixed point theorem was given by Jaggi [13] which involved a continuous map satisfying certain inequality involving rational expression. Kannan's study of fixed point theory involving uniformly convex Banach space and strictly Banach space was improved and sharpened by Jaggi [14] in the same year. Sharma and Yuel [27] obtained a unique fixed point by taking a continuous self mapping satisfying a rational type contraction in a complete metric space. There exists an extensive literature on fixed point theorems for various contractive conditions whose comprehensive survey can be found in Paliwal [20], Pathak [23], Reich [24], Rhoades [25], Smart [29] and Hussain et al. [12]. A unique common fixed point for a pair of continuous self mappings or continuous non self mappings studied by Bajaj [1], Chatterji [6], Fisher [9], Ganguly and Bandyopadhay [10]. Wong [31] obtained fixed point by replacing the constants by suitable non negative real valued functions. Common fixed point for a sequence of mappings obtained by Nadler [19]. A fixed point for a non continuous self map satisfying an integral type contraction was given by Gairola and Rawat [11].

The Banach principle has been extended and generalized by several authors in various spaces such as quasi-metric spaces, b-metric spaces, convex Banach spaces, G-metric spaces, cone metric spaces, partial ordered metric spaces, normed spaces by Koparde and Waghmode [18], Pandhare and Waghmode [21], Pandhare [22], Seshagiri Rao et al. [26], Veerapandi et al. [30] and so on.

The main aim of this paper is to investigate the existence and uniqueness of a common fixed point for a pair of continuous self mapping $T_{1}, T_{2}$, some positive integers powers $p, q$ of a pair of continuous self mappings $T_{1}^{p}, T_{2}^{q}$ and then further generalized to a sequence of continuous self mappings in the space. These results generalize and extend the results of $[3,7,15,17,21,24,26,28]$ in the literature.

\section{Main Results}

We start this section with the following theorem.

Theorem 2.1. Let $X$ be a closed subset of a Hilbert space and $T_{1}, T_{2}$ be two continuous self mappings on $X$ satisfying the following contraction condition, then $T_{1}$ and $T_{2}$ have a unique common fixed point in $X$ :

$$
\begin{aligned}
\left\|T_{1} x-T_{2} y\right\| & \leq a_{1} \frac{\left\|x-T_{1} x\right\|\left[1+\left\|y-T_{2} y\right\|\right]}{1+\|x-y\|}+a_{2} \frac{\left\|y-T_{2} y\right\|\left[1+\left\|y-T_{1} x\right\|\right]}{1+\|x-y\|} \\
& +a_{3} \frac{\left\|x-T_{2} y\right\|\left[1+\left\|y-T_{1} x\right\|\right]}{1+\|x-y\|}+a_{4} \frac{\|x-y\|\left[1+\left\|T_{1} x-T_{2} y\right\|\right]}{1+\|x-y\|}
\end{aligned}
$$




$$
\begin{aligned}
& +a_{5} \frac{\|x-y\|\left[1+\left\|x-T_{1} x\right\|\right]}{1+\left\|y-T_{2} y\right\|}+a_{6} \frac{\left\|x-T_{1} x\right\|\left[1+\left\|x-T_{2} y\right\|\right]}{1+\left\|y-T_{2} y\right\|} \\
& +a_{7} \frac{\left\|x-T_{2} y\right\|\left[1+\left\|T_{1} x-T_{2} y\right\|\right]}{1+\|x-y\|}+a_{8} \frac{\|x-y\|\left[1+\left\|x-T_{2} y\right\|\right]}{1+\|x-y\|} \\
& +a_{9} \frac{\left\|x-T_{1} x\right\|+\left\|y-T_{2} y\right\|+\|x-y\|}{1+\left\|x-T_{1} x\right\|\left\|x-T_{2} y\right\|\left\|y-T_{2} y\right\|\|x-y\|} \\
& +a_{10} \frac{\left\|x-T_{2} y\right\|^{2}+\left\|y-T_{1} x\right\|^{2}}{\left\|x-T_{2} y\right\|+\left\|y-T_{1} x\right\|}+a_{11}\left[\left\|x-T_{1} x\right\|+\left\|y-T_{2} y\right\|\right] \\
& +a_{12}\left[\left\|x-T_{2} y\right\|+\left\|y-T_{1} x\right\|\right]+a_{13}\|x-y\| .
\end{aligned}
$$

For all $x, y \in X$ and $x \neq y$, where $a_{i}(i=1,2,3, \ldots, 13)$ are non-negative reals with $0 \leq \sum_{i=1}^{8} a_{i}+3 a_{9}+2 \sum_{i=10}^{12} a_{i}+a_{13}<1$.

Proof. A sequence $\left\{x_{n}\right\}$ for an arbitrary point $x_{0} \in X$ defined as follows

$$
x_{2 n+1}=T_{1} x_{2 n}, x_{2 n+2}=T_{2} x_{2 n+1}, \text { for } n=0,1,2, \ldots .
$$

Now to show that the sequence $\left\{x_{n}\right\}$ is a Cauchy sequence in $X$ for that consider the following

$$
\begin{aligned}
\left\|x_{2 n+1}-x_{2 n}\right\| & =\left\|T_{1} x_{2 n}-T_{2} x_{2 n-1}\right\| \\
& \leq a_{1} \frac{\left\|x_{2 n}-T_{1} x_{2 n}\right\|\left[1+\left\|x_{2 n-1}-T_{2} x_{2 n-1}\right\|\right]}{1+\left\|x_{2 n}-x_{2 n-1}\right\|} \\
& +a_{2} \frac{\left\|x_{2 n-1}-T_{2} x_{2 n-1}\right\|\left[1+\left\|x_{2 n-1}-T_{1} x_{2 n}\right\|\right]}{1+\left\|x_{2 n}-x_{2 n-1}\right\|} \\
& +a_{3} \frac{\left\|x_{2 n}-T_{2} x_{2 n-1}\right\|\left[1+\left\|x_{2 n-1}-T_{1} x_{2 n}\right\|\right]}{1+\left\|x_{2 n}-x_{2 n-1}\right\|} \\
& +a_{4} \frac{\left\|x_{2 n}-x_{2 n-1}\right\|\left[1+\left\|T_{1} x_{2 n}-T_{2} x_{2 n-1}\right\|\right]}{1+\left\|x_{2 n}-x_{2 n-1}\right\|} \\
& +a_{5} \frac{\left\|x_{2 n}-x_{2 n-1}\right\|\left[1+\left\|x_{2 n}-T_{1} x_{2 n}\right\|\right]}{1+\left\|x_{2 n-1}-T_{2} x_{2 n-1}\right\|} \\
& +a_{6} \frac{\left\|x_{2 n}-T_{1} x_{2 n}\right\|\left[1+\left\|x_{2 n}-T_{2} x_{2 n-1}\right\|\right]}{1+\left\|x_{2 n-1}-T_{2} x_{2 n-1}\right\|} \\
& +a_{7} \frac{\left\|x_{2 n}-T_{2} x_{2 n-1}\right\|\left[1+\left\|T_{1} x_{2 n}-T_{2} x_{2 n-1}\right\|\right]}{1+\left\|x_{2 n}-x_{2 n-1}\right\|} \\
& +a_{8} \frac{\left\|x_{2 n}-x_{2 n-1}\right\|\left[1+\left\|x_{2 n}-T_{2} x_{2 n-1}\right\|\right]}{1+\left\|x_{2 n}-x_{2 n-1}\right\|}
\end{aligned}
$$




$$
\begin{aligned}
& +a_{9} \frac{\left\|x_{2 n}-T_{1} x_{2 n}\right\|+\left\|x_{2 n-1}-T_{2} x_{2 n-1}\right\|+\left\|x_{2 n}-x_{2 n-1}\right\|}{1+\left\|x_{2 n}-T_{1} x_{2 n}\right\|\left\|x_{2 n}-T_{2} x_{2 n-1}\right\|\left\|x_{2 n-1}-T_{2} x_{2 n-1}\right\|\left\|x_{2 n}-x_{2 n-1}\right\|} \\
& +a_{10} \frac{\left\|x_{2 n}-T_{2} x_{2 n-1}\right\|^{2}+\left\|x_{2 n-1}-T_{1} x_{2 n}\right\|^{2}}{\left\|x_{2 n}-T_{2} x_{2 n-1}\right\|+\left\|x_{2 n-1}-T_{1} x_{2 n}\right\|} \\
& +a_{11}\left[\left\|x_{2 n}-T_{1} x_{2 n}\right\|+\left\|x_{2 n-1}-T_{2} x_{2 n-1}\right\|\right] \\
& +a_{12}\left[\left\|x_{2 n}-T_{2} x_{2 n-1}\right\|+\left\|x_{2 n-1}-T_{1} x_{2 n}\right\|\right]+a_{13}\left\|x_{2 n}-x_{2 n-1}\right\|
\end{aligned}
$$

which implies that

$$
\left\|x_{2 n+1}-x_{2 n}\right\|=p(n)\left\|x_{2 n}-x_{2 n-1}\right\|,
$$

where

$$
p(n)=\frac{A_{1}+\left(a_{2}+2 a_{9}+a_{10}+a_{11}++a_{12}\right)\left\|x_{2 n}-x_{2 n-1}\right\|}{A_{2}+\left(1-a_{1}-a_{2}-a_{4}-a_{5}-a_{9}-a_{10}-a_{11}-a_{12}\right)\left\|x_{2 n}-x_{2 n-1}\right\|},
$$

and $A_{1}=a_{2}+a_{4}+a_{5}+a_{8}+2 a_{9}+a_{10}+a_{11}+a_{12}+a_{13}$ and $A_{2}=1-a_{1}-$ $a_{6}-a_{9}-a_{10}-a_{11}-a_{12}$.

Clearly, $\lambda=p(n)<1, \forall n=1,2,3, \ldots$, and hence in general, we get

$$
\left\|x_{n+1}-x_{n}\right\|=\lambda\left\|x_{n}-x_{n-1}\right\| \text {. }
$$

Continuing the above process, we get

$$
\left\|x_{n+1}-x_{n}\right\|=\lambda^{n}\left\|x_{1}-x_{0}\right\|, n \geq 1,
$$

taking $n \rightarrow \infty$, we obtain $\left\|x_{n+1}-x_{n}\right\| \rightarrow 0$. Hence, it follows that $\left\{x_{n}\right\}$ is a Cauchy sequence in $X$ and so it has a limit $\mu$ in $X$. Since the sequences $\left\{x_{2 n+1}\right\}=$ $\left\{T_{1} x_{2 n}\right\}$ and $\left\{x_{2 n+2}\right\}=\left\{T_{2} x_{2 n+1}\right\}$ are subsequences of $\left\{x_{n}\right\}$, and also these sub sequences have the same limit $\mu$ in $X$.

Next to show that $\mu$ is a common fixed point of $T_{1}$ and $T_{2}$. Now let us consider the following inequality

$$
\begin{aligned}
\left\|\mu-T_{1} \mu\right\| & =\left\|\left(\mu-x_{2 n+2}\right)+\left(x_{2 n+2}-T_{1} \mu\right)\right\| \\
& \leq\left\|\mu-x_{2 n+2}\right\|+\left\|T_{1} \mu-T_{2} x_{2 n+1}\right\| \\
& \leq a_{1} \frac{\left\|\mu-T_{1} \mu\right\|\left[1+\left\|x_{2 n+1}-T_{2} x_{2 n+1}\right\|\right]}{1+\left\|\mu-x_{2 n+1}\right\|} \\
& +a_{2} \frac{\left\|x_{2 n+1}-T_{2} x_{2 n+1}\right\|\left[1+\left\|x_{2 n+1}-T_{1} \mu\right\|\right]}{1+\left\|\mu-x_{2 n+1}\right\|} \\
& +a_{3} \frac{\left\|\mu-T_{2} x_{2 n+1}\right\|\left[1+\left\|x_{2 n+1}-T_{1} \mu\right\|\right]}{1+\left\|\mu-x_{2 n+1}\right\|} \\
& +a_{4} \frac{\left\|\mu-x_{2 n+1}\right\|\left[1+\left\|T_{1} \mu-T_{2} x_{2 n+1}\right\|\right]}{1+\left\|\mu-x_{2 n+1}\right\|}
\end{aligned}
$$




$$
\begin{aligned}
& +a_{5} \frac{\left\|\mu-x_{2 n+1}\right\|\left[1+\left\|\mu-T_{1} \mu\right\|\right]}{1+\left\|x_{2 n+1}-T_{2} x_{2 n+1}\right\|}+a_{6} \frac{\left\|\mu-T_{1} \mu\right\|\left[1+\left\|\mu-T_{2} x_{2 n+1}\right\|\right]}{1+\left\|x_{2 n+1}-T_{2} x_{2 n+1}\right\|} \\
& +a_{7} \frac{\left\|\mu-T_{2} x_{2 n+1}\right\|\left[1+\left\|T_{1} \mu-T_{2} x_{2 n+1}\right\|\right]}{1+\left\|\mu-x_{2 n+1}\right\|} \\
& +a_{8} \frac{\left\|\mu-x_{2 n+1}\right\|\left[1+\left\|\mu-T_{2} x_{2 n+1}\right\|\right]}{1+\left\|\mu-x_{2 n+1}\right\|} \\
& +a_{9} \frac{\left\|\mu-T_{1} \mu\right\|+\left\|x_{2 n+1}-T_{2} x_{2 n+1}\right\|+\left\|\mu-x_{2 n+1}\right\|}{1+\left\|\mu-T_{1} \mu\right\|\left\|\mu-T_{2} x_{2 n+1}\right\|\left\|x_{2 n+1}-T_{2} x_{2 n+1}\right\|\left\|\mu-x_{2 n+1}\right\|} \\
& +a_{10} \frac{\left\|\mu-T_{2} x_{2 n+1}\right\|^{2}+\left\|x_{2 n+1}-T \mu\right\|^{2}}{\left\|\mu-T_{2} x_{2 n+1}\right\|+\left\|x_{2 n+1}-T_{1} \mu\right\|} \\
& +a_{11}\left[\left\|\mu-T_{1} \mu\right\|+\left\|x_{2 n+1}-T_{2} x_{2 n+1}\right\|\right] \\
& +a_{12}\left[\left\|\mu-T_{2} x_{2 n+1}\right\|+\left\|x_{2 n+1}-T_{1} \mu\right\|\right]+a_{13}\left\|\mu-x_{2 n+1}\left|+\| \mu-x_{2 n+2}\right| .\right.
\end{aligned}
$$

Letting $n \rightarrow \infty$, we obtain $\left\|\mu-T_{1} \mu\right\| \leq\left(a_{1}+a_{6}+a_{9}+a_{10}+a_{11}+a_{12}\right)\left\|\mu-T_{1} \mu\right\|$, since $a_{1}+a_{6}+a_{9}+a_{10}+a_{11}+a_{12}<1$, it follows immediately that $T_{1} \mu=\mu$.

Similarly, from hypothesis, we can get $T_{2} \mu=\mu$ by considering the following

$$
\left\|\mu-T_{2} \mu\right\|=\left\|\left(\mu-x_{2 n+1}\right)+\left(x_{2 n+1}-T_{2} \mu\right)\right\| .
$$

Finally, we want to show that $\mu$ is a unique fixed point of $T_{1}, T_{2}$. Let us suppose that $v(\mu \neq v)$ is also a common fixed point of $T_{1}$ and $T_{2}$. Then, in view of hypothesis, we have

$$
\begin{aligned}
\|\mu-v\| & =\left\|T_{1} \mu-T_{2} v\right\| \\
& \leq a_{1} \frac{\left\|\mu-T_{1} \mu\right\|\left[1+\left\|v-T_{2} v\right\|\right]}{1+\|\mu-v\|}+a_{2} \frac{\left\|v-T_{2} v\right\|\left[1+\left\|v-T_{1} \mu\right\|\right]}{1+\|\mu-v\|} \\
& +a_{3} \frac{\left\|\mu-T_{2} v\right\|\left[1+\left\|v-T_{1} \mu\right\|\right]}{1+\|\mu-v\|}+a_{4} \frac{\|\mu-v\|\left[1+\left\|T_{1} \mu-T_{2} v\right\|\right]}{1+\|\mu-v\|} \\
& +a_{5} \frac{\|\mu-v\|\left[1+\left\|\mu-T_{1} \mu\right\|\right]}{1+\left\|v-T_{2} v\right\|}+a_{6} \frac{\left\|\mu-T_{1} \mu\right\|\left[1+\left\|\mu-T_{2} v\right\|\right]}{1+\left\|v-T_{2} v\right\|} \\
& +a_{7} \frac{\left\|\mu-T_{2} v\right\|\left[1+\left\|T_{1} \mu-T_{2} v\right\|\right]}{1+\|\mu-v\|}+a_{8} \frac{\|\mu-v\|\left[1+\left\|\mu-T_{2} v\right\|\right]}{1+\|\mu-v\|} \\
& +a_{9} \frac{\left\|\mu-T_{1} \mu\right\|+\left\|v-T_{2} v\right\|+\|\mu-v\|}{1+\left\|\mu-T_{1} \mu\right\|\left\|\mu-T_{2} v\right\|\left\|v-T_{2} v\right\|\|\mu-v\|} \\
& +a_{10} \frac{\left\|\mu-T_{2} v\right\|^{2}+\left\|v-T_{1} \mu\right\|^{2}}{\left\|\mu-T_{2} v\right\|+\left\|v-T_{1} \mu\right\|}+a_{11}\left[\left\|\mu-T_{1} \mu\right\|+\left\|v-T_{2} v\right\|\right] \\
& +a_{12}\left[\left\|\mu-T_{2} v\right\|+\left\|v-T_{1} \mu\right\|\right]+a_{13}\|\mu-v\| .
\end{aligned}
$$


Thus,

$\|\mu-v\| \leq\left(a_{3}+a_{4}+a_{5}+a_{7}+a_{8}+a_{9}+a_{10}+2 a_{12}+a_{13}\right)\|\mu-v\|<\|\mu-v\|$

which is a contradiction and hence it follows that $\mu=v$. So, the common fixed point $\mu$ is unique in $X$.

Corollary 2.2. Let $X$ be a closed subset of a Hilbert space and $T: X \rightarrow X$ be a continuous self mapping satisfying the following inequality

$$
\begin{aligned}
\|T x-T y\| & \leq a_{1} \frac{\|x-T x\|[1+\|y-T y\|]}{1+\|x-y\|}+a_{2} \frac{\|y-T y\|[1+\|y-T x\|]}{1+\|x-y\|} \\
& +a_{3} \frac{\|x-T y\|[1+\|y-T x\|]}{1+\|x-y\|}+a_{4} \frac{\|x-y\|[1+\|T x-T y\|]}{1+\|x-y\|} \\
& +a_{5} \frac{\|x-y\|[1+\|x-T x\|]}{1+\|y-T y\|}+a_{6} \frac{\|x-T x\|[1+\|x-T y\|]}{1+\|y-T y\|} \\
& +a_{7} \frac{\|x-T y\|[1+\|T x-T y\|]}{1+\|x-y\|}+a_{8} \frac{\|x-y\|[1+\|x-T y\|]}{1+\|x-y\|} \\
& +a_{9} \frac{\|x-T x\|+\|y-T y\|+\|x-y\|}{1+\|x-T x\|\|x-T y\|\|y-T y\|\|x-y\|} \\
& +a_{10} \frac{\|x-T y\|^{2}+\|y-T x\|^{2}}{\|x-T y\|+\|y-T x\|}+a_{11}[\|x-T x\|+\|y-T y\|] \\
& +a_{12}[\|x-T y\|+\|y-T x\|]+a_{13}\|x-y\|
\end{aligned}
$$

for all $x, y \in X$ and $x \neq y$, where $a_{i}(i=1,2,3, \ldots, 13)$ are non-negative reals with $0 \leq \sum_{i=1}^{8} a_{i}+3 a_{9}+2 \sum_{i=10}^{12} a_{i}+a_{13}<1$. Then $T$ has a unique fixed point in $X$.

Proof. Set $T_{1}=T_{2}=T$ in Theorem 2.1 .

Remark 2.3. The following results are obtained from the above Corollary 2.2 by taking variations in the variables, making some of the real constants to zeros and restricting the space from Hilbert space to metric space.

(i) Kannan [17] type mapping in Hilbert space can get by taking $a_{i}=0, i=$ $1,2,3, \ldots, 10,12,13$ in Corollary 2.2.

(ii) The extended Kannan [17] type condition in Hilbert space can obtain by replacing $a_{i}=0, i=1,2,3, \ldots, 10,12$ in Corollary 2.2 . 
(iii) Chatterjee [3] contraction condition in Hilbert space can get by putting $a_{i}=$ $0, i=1,2,3, \ldots, 11,13$ in Corollary 2.2.

(iv) The extended contraction type condition of Chatterjee [3] in Hilbert space can get by giving $a_{i}=0, i=1,2,3, \ldots, 11$ in Corollary 2.2.

(v) Sharm et al. [28] type contraction can obtain from Corollary 2.2 by replacing $a_{i}=0, i=1,2,3, \ldots, 9,11,12$.

(vi) Taking variations in variables and putting $a_{4}=a_{5}=a_{6}=a_{7}=a_{8}=$ $0, a_{11}=\beta$, we obtain the result of [15].

(vii) Dass and Gupta [7] result can be found on taking $a_{i}=0(i=2,3, \ldots, 10)$ in restricting the Hilbert space to the metric space and taking the variations in the variables.

(viii) A comparison reveals that Corollary 2.2 reduces to [26] on taking $a_{2}=a_{4}=$ $a_{5}=a_{6}=a_{7}=a_{8}=a_{9}=a_{10}=0$.

Theorem 2.4. Let $X$ be a closed subset of a Hilbert space and let $T_{1}, T_{2}$ be two continuous self mappings on $X$ satisfying

$$
\begin{aligned}
& \left\|T_{1}^{p} x-T_{2}^{q} y\right\| \leq a_{1} \frac{\left\|x-T_{1}^{p} x\right\|\left[1+\left\|y-T_{2}^{q} y\right\|\right]}{1+\|x-y\|} \\
& +a_{2} \frac{\left\|y-T_{2}^{q} y\right\|\left[1+\left\|y-T_{1}^{p} x\right\|\right]}{1+\|x-y\|}+a_{3} \frac{\left\|x-T_{2}^{q} y\right\|\left[1+\left\|y-T_{1}^{p} x\right\|\right]}{1+\|x-y\|} \\
& +a_{4} \frac{\|x-y\|\left[1+\left\|T_{1}^{p} x-T_{2}^{q} y\right\|\right]}{1+\|x-y\|}+a_{5} \frac{\|x-y\|\left[1+\left\|x-T_{1}^{p} x\right\|\right]}{1+\left\|y-T_{2}^{q} y\right\|} \\
& +a_{6} \frac{\left\|x-T_{1}^{p} x\right\|\left[1+\left\|x-T_{2}^{q} y\right\|\right]}{1+\left\|y-T_{2}^{q} y\right\|}+a_{7} \frac{\left\|x-T_{2}^{q} y\right\|\left[1+\left\|T_{1}^{p} x-T_{2}^{q} y\right\|\right]}{1+\|x-y\|} \\
& \quad+a_{8} \frac{\|x-y\|\left[1+\left\|x-T_{2}^{q} y\right\|\right]}{1+\|x-y\|} \\
& \quad+a_{9} \frac{\left\|x-T_{1}^{p} x\right\|+\left\|y-T_{2}^{q} y\right\|+\|x-y\|}{1+\left\|x-T_{1}^{p} x\right\|\left\|x-T_{2}^{q} y\right\|\left\|y-T_{2}^{q} y\right\|\|x-y\|} \\
& \quad+a_{10} \frac{\left\|x-T_{2}^{q} y\right\|^{2}+\left\|y-T_{1}^{p} x\right\|^{2}}{\left\|x-T_{2}^{q} y\right\|+\left\|y-T_{1}^{p} x\right\|}+a_{11}\left[\left\|x-T_{1}^{p} x\right\|+\left\|y-T_{2}^{q} y\right\|\right] \\
& \quad+a_{12}\left[\left\|x-T_{2}^{q} y\right\|+\left\|y-T_{1}^{p} x\right\|\right]+a_{13}\|x-y\|
\end{aligned}
$$

for all $x, y \in X$ and $x \neq y$, where $a_{i}(i=1,2,3, \ldots, 13)$ are non-negative reals with $0 \leq \sum_{i=1}^{8} a_{i}+3 a_{9}+2 \sum_{i=10}^{12} a_{i}+a_{13}<1$ and $p$, $q$ are two positive integers. Then $T_{1}$ and $T_{2}$ have a unique common fixed point in $X$. 
Proof. From Theorem 2.1, $T_{1}^{p}$ and $T_{2}^{q}$ have a unique common fixed point $\mu \in X$, so that $T_{1}^{p} \mu=\mu$ and $T_{2}^{q} \mu=\mu$.

From $T_{1}^{p}\left(T_{1} \mu\right)=T_{1}\left(T_{1}^{p} \mu\right)=T_{1} \mu$, it follows that $T_{1} \mu$ is a fixed point of $T_{1}^{p}$. But $\mu$ is a unique fixed point of $T_{1}^{p}$. Therefore $T_{1} \mu=\mu$. Similarly, we can get $T_{2} \mu=\mu$. Hence, $\mu$ is a common fixed point of $T_{1}$ and $T_{2}$. For uniqueness, let $v$ be another fixed point of $T_{1}$ and $T_{2}$, so that $T_{1} v=T_{2} v=v$. Then from hypothesis, we have

$$
\begin{aligned}
\|\mu-v\| & =\left\|T_{1}^{p} \mu-T_{2}^{q} v\right\| \\
& \leq a_{1} \frac{\left\|\mu-T_{1}^{p} \mu\right\|\left[1+\left\|v-T_{2}^{q} v\right\|\right]}{1+\|\mu-v\|}+a_{2} \frac{\left\|v-T_{2}^{q} v\right\|\left[1+\left\|v-T_{1}^{p} \mu\right\|\right]}{1+\|\mu-v\|} \\
& +a_{3} \frac{\left\|\mu-T_{2}^{q} v\right\|\left[1+\left\|v-T_{1}^{p} \mu\right\|\right]}{1+\|\mu-v\|}+a_{4} \frac{\|\mu-v\|\left[1+\left\|T_{1}^{p} \mu-T_{2}^{q} v\right\|\right]}{1+\|\mu-v\|} \\
& +a_{5} \frac{\|\mu-v\|\left[1+\left\|\mu-T_{1}^{p} \mu\right\|\right]}{1+\left\|v-T_{2}^{q} v\right\|}+a_{6} \frac{\left\|\mu-T_{1}^{p} \mu\right\|\left[1+\left\|\mu-T_{2}^{q} v\right\|\right]}{1+\left\|v-T_{2}^{q} v\right\|} \\
& +a_{7} \frac{\left\|\mu-T_{2}^{q} v\right\|\left[1+\left\|T_{1}^{p} \mu-T_{2}^{q} v\right\|\right]}{1+\|\mu-v\|}+a_{8} \frac{\|\mu-v\|\left[1+\left\|\mu-T_{2}^{q} v\right\|\right]}{1+\|\mu-v\|} \\
& +a_{9} \frac{\left\|\mu-T_{1}^{p} \mu\right\|+\left\|v-T_{2}^{q} v\right\|+\|\mu-v\|}{1+\left\|\mu-T_{1}^{p} \mu\right\|\left\|\mu-T_{2}^{q} v\right\|\left\|v-T_{2}^{q} v\right\|\|\mu-v\|} \\
& +a_{10} \frac{\left\|\mu-T_{2}^{q} v\right\|^{2}+\left\|v-T_{1}^{p} \mu\right\|^{2}}{\left\|\mu-T_{2}^{q} v\right\|+\left\|v-T_{1}^{p} \mu\right\|}+a_{11}\left[\left\|\mu-T_{1}^{p} \mu\right\|+\left\|v-T_{2}^{q} v\right\|\right] \\
& +a_{12}\left[\left\|\mu-T_{2}^{q} v\right\|+\left\|v-T_{1}^{p} \mu\right\|\right]+a_{13}\|\mu-v\| \\
\Rightarrow \| \mu & -v\left\|\leq\left(a_{3}+a_{4}+a_{5}+a_{7}+a_{8}+a_{9}+a_{10}+2 a_{12}+a_{13}\right)\right\| \mu-v \| \\
\Rightarrow \mu & =v, \text { since } a_{3}+a_{4}+a_{5}+a_{7}+a_{8}+a_{9}+a_{10}+2 a_{12}+a_{13}<1 .
\end{aligned}
$$

Hence, $\mu$ is a unique common fixed point of $T_{1}$ and $T_{2}$ in $X$.

This completes the proof of the theorem.

In the upcoming theorem, we have taken a sequence of continuous self mappings on a closed subset of a Hilbert space converging point wise to a limit mapping and show that if this limit mapping has a fixed point then this fixed point is also the limit of fixed points of the mappings of the sequence.

Theorem 2.5. Let $X$ be a closed subset of a Hilbert space and let $\left\{T_{i}\right\}$ be a sequence of continuous self mappings on $X$ converging point wise to a continuous 
map $T$ and let

$$
\begin{aligned}
\left\|T_{i} x-T_{i} y\right\| & \leq a_{1} \frac{\left\|x-T_{i} x\right\|\left[1+\left\|y-T_{i} y\right\|\right]}{1+\|x-y\|}+a_{2} \frac{\left\|y-T_{i} y\right\|\left[1+\left\|y-T_{i} x\right\|\right]}{1+\|x-y\|} \\
& +a_{3} \frac{\left\|x-T_{i} y\right\|\left[1+\left\|y-T_{i} x\right\|\right]}{1+\|x-y\|}+a_{4} \frac{\|x-y\|\left[1+\left\|T_{i} x-T_{i} y\right\|\right]}{1+\|x-y\|} \\
& +a_{5} \frac{\|x-y\|\left[1+\left\|x-T_{i} x\right\|\right]}{1+\left\|y-T_{i} y\right\|}+a_{6} \frac{\left\|x-T_{i} x\right\|\left[1+\left\|x-T_{i} y\right\|\right]}{1+\left\|y-T_{i} y\right\|} \\
& +a_{7} \frac{\left\|x-T_{i} y\right\|\left[1+\left\|T_{i} x-T_{i} y\right\|\right]}{1+\|x-y\|}+a_{8} \frac{\|x-y\|\left[1+\left\|x-T_{i} y\right\|\right]}{1+\|x-y\|} \\
& +a_{9} \frac{\left\|x-T_{i} x\right\|+\left\|y-T_{i} y\right\|+\|x-y\|}{1+\left\|x-T_{i} x\right\|\left\|x-T_{i} y\right\|\left\|y-T_{i} y\right\|\|x-y\|} \\
& +a_{10} \frac{\left\|x-T_{i} y\right\|^{2}+\left\|y-T_{i} x\right\|^{2}}{\left\|x-T_{i} y\right\|+\left\|y-T_{i} x\right\|}+a_{11}\left[\left\|x-T_{i} x\right\|+\left\|y-T_{i} y\right\|\right] \\
& +a_{12}\left[\left\|x-T_{i} y\right\|+\left\|y-T_{i} x\right\|\right]+a_{13}\|x-y\|
\end{aligned}
$$

for all $x, y \in X$ and $x \neq y$, where $a_{i}(i=1,2,3, \ldots, 13)$ are non-negative reals with $0 \leq \sum_{i=1}^{8} a_{i}+3 a_{9}+2 \sum_{i=10}^{12} a_{i}+a_{13}<1$. If each $T_{i}$ has a fixed point $\mu_{i}$ and $T$ has a fixed point $\mu$, then the sequence $\left\{\mu_{i}\right\}$ converges to $\mu$.

Proof. Since $\mu_{i}$ is a fixed point of $T_{i}$, then we have

$$
\begin{aligned}
& \left\|\mu-\mu_{n}\right\|=\left\|T \mu-T_{n} \mu_{n}\right\| \\
& =\left\|\left(T \mu-T_{n} \mu\right)+\left(T_{n} \mu-T_{n} \mu_{n}\right)\right\| \leq\left\|T \mu-T_{n} \mu\right\|+\left\|T_{n} \mu-T_{n} \mu_{n}\right\| \\
& \leq a_{1} \frac{\left\|\mu-T_{n} \mu\right\|\left[1+\left\|\mu_{n}-T_{n} \mu_{n}\right\|\right]}{1+\left\|\mu-\mu_{n}\right\|}+a_{2} \frac{\left\|\mu_{n}-T_{n} \mu_{n}\right\|\left[1+\left\|\mu_{n}-T_{n} \mu\right\|\right]}{1+\left\|\mu-\mu_{n}\right\|} \\
& +a_{3} \frac{\left\|\mu-T_{n} \mu_{n}\right\|\left[1+\left\|\mu_{n}-T_{n} \mu\right\|\right]}{1+\left\|\mu-\mu_{n}\right\|}+a_{4} \frac{\left\|\mu-\mu_{n}\right\|\left[1+\left\|T_{n} \mu-T_{n} \mu_{n}\right\|\right]}{1+\left\|\mu-\mu_{n}\right\|} \\
& +a_{5} \frac{\left\|\mu-\mu_{n}\right\|\left[1+\left\|\mu-T_{n} \mu\right\|\right]}{1+\left\|\mu_{n}-T_{n} \mu_{n}\right\|}+a_{6} \frac{\left\|\mu-T_{n} \mu\right\|\left[1+\left\|\mu-T_{n} \mu_{n}\right\|\right]}{1+\left\|\mu_{n}-T_{n} \mu_{n}\right\|} \\
& +a_{7} \frac{\left\|\mu-T_{n} \mu_{n}\right\|\left[1+\left\|T_{n} \mu-T_{n} \mu_{n}\right\|\right]}{1+\left\|\mu-\mu_{n}\right\|}+a_{8} \frac{\left\|\mu-\mu_{n}\right\|\left[1+\left\|\mu-T_{n} \mu_{n}\right\|\right]}{1+\left\|\mu-\mu_{n}\right\|} \\
& +a_{9} \frac{\left\|\mu-T_{n} \mu\right\|+\left\|\mu_{n}-T_{n} \mu_{n}\right\|+\left\|\mu-\mu_{n}\right\|}{1+\left\|\mu-T_{n} \mu\right\|\left\|\mu-T_{n} \mu_{n}\right\|\left\|\mu_{n}-T_{n} \mu_{n}\right\|\left\|\mu-\mu_{n}\right\|} \\
& +a_{10} \frac{\left\|\mu-T_{n} \mu_{n}\right\|{ }^{2}+\left.\left\|\mu_{n}-T_{n} \mu\right\|\right|^{2}}{\left\|\mu-T_{n} \mu_{n}\right\|+\left\|\mu_{n}-T_{n} \mu\right\|}+a_{11}\left[\left\|\mu-T_{n} \mu\right\|+\left\|\mu_{n}-T_{n} \mu_{n}\right\|\right] \\
& +a_{12}\left[\left\|\mu-T_{n} \mu_{n}\right\|+\left\|\mu_{n}-T_{n} \mu\right\|\right]+a_{13}\left\|\mu-\mu_{n}\right\|+\left\|T \mu-T_{n} \mu\right\| .
\end{aligned}
$$


Letting $n \rightarrow \infty$, so that $T_{n} \mu \rightarrow T \mu, T_{n} \mu_{n} \rightarrow \mu_{n}$ and $T \mu=\mu$, we get

$\lim _{n \rightarrow \infty}\left\|\mu-\mu_{n}\right\| \leq\left(a_{4}+a_{5}+a_{7}+a_{8}+a_{9}+a_{10}+2 a_{12}+a_{13}\right) \lim _{n \rightarrow \infty}\left\|\mu-\mu_{n}\right\|$.

So, $\lim _{n \rightarrow \infty}\left\|\mu-\mu_{n}\right\|=0$, since $a_{4}+a_{5}+a_{7}+a_{8}+a_{9}+a_{10}+2 a_{12}+a_{13}<1$. Thus, $\mu_{n} \rightarrow \mu$ as $n \rightarrow \infty$. This completes the proof.

Example 2.6. The following is an example of Theorem 2.1:

Let $T_{1}, T_{2}:[0,1] \rightarrow[0,1]$ be defined as $T_{1} x=\frac{x}{2}$ and $T_{2} x=\frac{x}{4}$, for all $x \in[0,1]$. Then with usual norm $\|x-y\|=|x-y|$ one can see that 0 is the only common fixed point of $T_{1}$ and $T_{2}$.

Example 2.7. For an example of Corollary 2.2, let $T:[0,1] \rightarrow[0,1]$ be a mapping defined by $T x=\frac{x^{3}}{6}$, for all $x \in[0,1]$. Obviously, 0 is the only fixed point of $T$ with usual norm $\|x-y\|=|x-y|$, for all $x \in[0,1]$.

\section{Conclusions}

The Banach contraction principle has been refined and extended on a closed subset of a Hilbert space to a pair of continuous self mappings involving more number of rational terms in the contractive condition. The same result is extended for the positive integers powers of a pair of continuous self mappings and then further developed to a sequence of continuous self mappings in the space. In all different cases, we have observed the existence and uniqueness of a common fixed point in the space. From these results we have obtained well known results in the literature at particular cases.

Acknowledgments. We would like to thank the referees for carefully reading of our manuscript and for their valuable suggestions and remarks which improve the strength of paper.

\section{Bibliography}

[1] N. Bajaj, Some maps on unique common fixed points, Indian J. Pure. App. Math. 15(8) (1984), 843-848.

[2] S. Banach, Sur les operations dans les ensembles abstraits et leur application aux equations untegrales, Fund. Math. 3 (1922), 133-181.

[3] S. K. Chetterjee, Fixed Point Theorems, C. R. Acad. Bulgara Sci. 25 (1972), 727730 . 
[4] H. Chatterji, On generalization of Banach contraction principle, Indian J. Pure. App. Math. 10(4) (1979), 400-403.

[5] H. Chatterji, A fixed point theorem in metric spaces, Indian J. Pure. App. Math. 10(4) (1979), 449-450.

[6] H. Chatterji, Fixed points for a pair of mappings, Indian J. Pure. App. Math. 10(7) (1979), 886-889.

[7] B. K. Dass and S. Gupta, An extension of Banach contraction principle through rational expression, Indian J. Pure. App. Math. 6 (1975), 1455-1458.

[8] B. Fisher, Fixed point mappings, Atti. Della. Acad. Naz. De Lincei LIX, Fasc. 5 (1975).

[9] B. Fisher, Common fixed point and constant mappings satisfying a rational inequality, Math. Sem. Notes 6 (1978), 29-35.

[10] D. K. Ganguly and D. Bandyopadhyay, Some results on common fixed point theorems in metric space, Bull. Cal. Math. Soc. 83 (1991), 137-145.

[11] U. C. Gairola and A. S. Rawat, A fixed point theorem for non-continuous maps satisfying integral type inequality, J. Indian Math. Soc. (N.S.) 80 (2013), 69-77.

[12] N. Hussain, D. Doric, Z. Kadelburg and S. Radenovic, Suzuki-type fixed point results in metric type spaces, Fixed Point Theory Appl. 2012 (2012), Article ID 126.

[13] D. S. Jaggi, Some unique fixed point theorems, Indian J. Pure. App. Math. 8(2) (1977), 223-230.

[14] D. S. Jaggi, Fixed point theorems for orbitally continuous function-II, Indian J. Math. 19(2) (1977), 113-118.

[15] K. Kalyani, N. Seshagiri Rao and K. V. L. N. Acharyulu, A fixed point of self mapping in Hilbert space, Global Journal of Pure and Applied Mathematics 10(6) (2014), 783-786.

[16] R. Kannan, Some results on fixed points, Bull. Cal. Math. Soc. 60 (1968), 71-76.

[17] R. Kannan, Some results on fixed points-II, Amer. Math. Monthly 76 (1969), 405408.

[18] P. V. Koparde and B. B. Waghmode, On sequence of mappings in Hilbert space, The Mathematics Education 25(4) (1991), 197-198.

[19] S. B. Nadler, Sequence of contraction and fixed points, Pacific J. Math., 27 (1968), 579-585.

[20] Y. C. Paliwal, Fixed point theorem in metric space, Bull. Cal. Math. Soc. 80 (1988), 205-208.

[21] D. M. Pandhare and B. B. Waghmode, Fixed point theorems for the extension of Kannan's type mappings in Hilbert spaces, The Mathematics Education 28(4) (1994), 189-193. 
[22] D. M. Pandhare, On the sequence of mappings on Hilbert space, The Mathematics Education 32(2) (1998), 61-63.

[23] H. K. Pathak, Some fixed point theorems on contractive mappings, Bull. Cal. Math. Soc. 80 (1988), 183-188.

[24] S. Reich, Some remarks concerning contraction mappings, Canad. Math. Bull. 14 (1971), 121-124.

[25] B. E. Rhoades, A comparison of various definitions of contractive mappings, Trans. Amer. Math. Soc. 226 (1977), 257-290.

[26] N. Seshagiri Rao, K. Kalyani and K. V. L. N. Acharyulu, A unique fixed point theorem in Hilbert space, Acta Ciencia Indica XLI(1) (2015), 39-46.

[27] P. L. Sharma and A. K. Yuel, A unique fixed point theorem in metric space, Bull. Cal. Math. Soc. 76 (1984), 153-156.

[28] A. K. Sharma, V. H. Babshah and V. K. Gupta, Common fixed point theorems of a sequence of mappings in Hilbert spaces, Journal of Ultra Scientist Phyl. Sci. 23(3A) (2012), 790-794.

[29] D. R. Smart, Fixed Point Theorems, Cambridge University Press, 1974.

[30] T. Veerapandi and S. Anil Kumar, Common fixed point theorems of a sequence of mappings on Hilbert space, Bull. Cal. Math. Soc. 91(4) (1999), 299-308.

[31] C. S. Wong, Common fixed points of two mappings, Pacific J. Math. 48 (1973), 299-312.

[32] T. Zamfirescu, Fixed point theorems in metric spaces, Arch. Math. 23 (1972), 292298.

Received November 13, 2019; revised March 12, 2020; accepted March 28, 2020.

\section{Author information}

Namana Seshagiri Rao, Department of Applied Mathematics, School of Applied Natural Science, Adama Science and Technology University, Post Box No.1888, Adama,

Ethiopia.

E-mail: seshu.namana@gmail.com

Karusala Kalyani, Department of Mathematics, Vignan's Foundation for Science, Technology \& Research, Vadlamudi-522213, Andhra Pradesh, India.

E-mail: kalyani.namana@gmail.com 